\title{
Percepción de actores educativos acerca del uso de dispositivos móviles: un estudio de caso
}

\section{Perception of educational actors about the use of mobile devices: A case study}

\author{
Verónica Maldonado-Garcés \\ Pontificia Universidad Católica del Ecuador \\ vmaldonado794@puce.edu.ec \\ https://orcid.org/0000-0002-4853-2239 \\ Jorge Balladares-Burgos \\ Pontificia Universidad Católica del Ecuador \\ jballadares@puce.edu.ec \\ https://orcid.org/0000-0001-7033-1970 \\ Alex Rivas -Toledo \\ Pontificia Universidad Católica del Ecuador \\ avrivas@puce.edu.ec \\ https://orcid.org/0000-0001-9299-8338
}

(Recibido: 10/09/2019; Aceptado: 12/09/2019; Versión final recibida: 16/09/2019)

Cita del artículo: Maldonado-Garcés, V., Balladares-Burgos, J., Rivas-Toledo, A., (2019). Percepción de actores educativos acerca del uso de dispositivos móviles: un estudio de caso. Revista Cátedra, 2(3), 39-53.

\section{Resumen}

La implementación de metodologías innovadoras relacionadas con el uso de recursos digitales ha permitido una participación relevante de las comunidades educativas. Este artículo da cuenta de las miradas de los estudiantes y docentes de una institución educativa fiscal beneficiaria del Proyecto Aula Digital Móvil ejecutada por Fundación Telefónica en el marco del Proyecto Profuturo y articulada con el Ministerio de Educación del Ecuador.

La investigación pretende determinar la percepción tanto de los docentes como de los estudiantes con respecto al uso educativo de dispositivos móviles. Se empleó el método inductivo, de manera que el punto de partida fueron los datos proporcionados por los 70 niños en edad escolar y 10 docentes para realizar la construcción de las categorías.

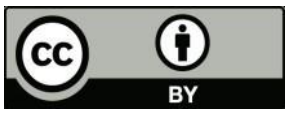


El 75\% de los participantes de esta investigación asocian el uso del dispositivo móvil con el aprendizaje, refiriéndose además a un aprendizaje adquirido de forma divertida. Además, mencionan que la tableta les permite acceder a información, conocer otras culturas, posibilita la investigación lo que genera un aprendizaje significativo.

El dispositivo móvil dentro del aula ha logrado resultados positivos en los procesos de aprendizaje, la totalidad de los participantes perciben y valoran el recurso como positivo, lo asumen como un puente que les acerca al conocimiento de otras culturas, les da la posibilidad de investigar, de aprender valores, contenidos de asignaturas y de acceder a la lectura.

\section{Palabras clave}

Aprendizaje, dispositivos móviles, percepción, tabletas.

\section{Abstract}

The implementation of innovative methodologies related to the use of digital resources has allowed a relevant participation of educational communities. This article provides the views of the students and teachers of an educational institution benefiting from the Mobile Digital Classroom Project executed by Fundación Telefónica, part of the Profuturo Project and articulated with the Ministry of Education of Ecuador. The research aims to determine the perception of teachers and students in relation to the educational use of mobile devices. The inductive method was used, thus, the starting point was the data provided by the 70 children of school age and 10 teachers to perform the construction of the categories.

$75 \%$ of the participants of this research associate the use of the mobile device with learning, also referring to the learning acquired in a fun way. In addition, they mention that the tablet allows them to access information, to know other cultures, to enable research, which generates significant learning.

The mobile device within the classroom achieved positive results in the learning processes, all participants perceive and value the resource as positive, they assume it as a bridge that brings them closer to the knowledge of other cultures, gives them the possibility to investigate, to learn values, content of topics and access to reading.

\section{Keywords}

Learning, mobile devices, perception, tablets.

\section{Introducción}

En el marco del Plan Nacional del Buen Vivir 2017-2021 y los Nueve Objetivos de Desarrollo del Ecuador, el Ministerio de Educación del Ecuador presentó en 2017 la Agenda Educativa Digital (2017 - 2021) cuyo objetivo es "fortalecer y potenciar el proceso de enseñanzaaprendizaje en el Sistema Educativo Nacional a través del incremento de prácticas innovadoras que integren las tecnologías para empoderar el aprendizaje, el conocimiento y la participación" (2019, pág. 2).

La Agenda Educativa Digital está basada en una estructura compuesta por cinco ejes: Eje físico, Eje de aprendizaje digital, Eje de desarrollo docente, Eje de comunicación y fomento y, finalmente, Eje de innovación. De acuerdo con el Enfoque de la Agenda Educativa Digital, el quinto eje sobre innovación plantea al aula digital móvil como un proyecto de transformación pedagógica que busca la inserción de la tecnología en el aula. 
Articulada a estos planteamientos, Fundación Telefónica, en el marco de la Propuesta Profuturo, implementa el Proyecto Aula Digital Móvil en 70 escuelas públicas del Ecuador para beneficiar a alrededor de 12.600 niños y 2.100 docentes. Así, es meritorio estudiar la percepción de los actores educativos acerca del uso de dispositivos móviles. Esto con el objeto de revisar si docentes y estudiantes aceptan o rechazan el uso de la tecnología en el aula.

Además, otro objetivo consiste en analizar la usabilidad de los recursos en el marco de los procesos de enseñanza aprendizaje. Este tema cobra importancia al relacionarse con la calidad educativa. El uso de la tecnología podría constituirse en un factor decisivo capaz de lograr el aprendizaje significativo en los educandos. Por ello hemos analizado las percepciones de los docentes y estudiantes acerca de la implementación del Proyecto Aula Digital Profuturo. Por otro lado se categorizaron las percepciones y se relacionaron estos resultados entre docentes y estudiantes en edad escolar.

Para el efecto de este estudio es importante revisar el objetivo del Proyecto Aula Digital Móvil, el mismo que pretende:

Contribuir a la adquisición de competencias de niños y niñas, a través de la tecnología, y el empoderamiento de los docentes para la aplicación de metodologías innovadoras de enseñanza (Fundación Telefónica, 2017, pág. 1).

La institución educativa pública participante de esta investigación recibió por parte de la Fundación Telefónica un conjunto de recursos tecnológicos. Estos consistieron en una computadora, tabletas, un concentrador de carga, un router wifi, un mini proyector y una regleta. Además, el Aula Digital Móvil, a través del dispositivo tableta, dispone de varios recursos a los que los estudiantes pueden acceder bajo la guía del docente. El aula digital ofrece una base de contenidos y actividades digitales dispuestas en su plataforma: weclass.

La capacitación y el acompañamiento a docentes y autoridades de las escuelas donde se implementó el proyecto es un eje importante dentro de la implementación del aula móvil digital. Los docentes recibieron capacitaciones previas a la ejecución del Proyecto. Se realizaron cursos bajo la modalidad presencial mediante la metodología de clase inversa y también la formación se efectuó a través de una plataforma virtual weclass.

Todas estas acciones nos ubican en el área del aprendizaje digital. Este hace referencia a las nuevas prácticas de enseñanza y aprendizajes propios de la era digital. Su objetivo, para el MINEDUC, es desarrollar en los estudiantes y los docentes el pensamiento crítico, la creatividad y el protagonismo en la solución de problemas de la vida. Estas estrategias deben vincularse a su entorno, mediante el uso de objetos digitales virtualizados de manera lúdica, visual, intuitiva. Para que esto sea posible, un factor decisivo constituye la percepción de docentes y estudiantes frente al aprendizaje digital.

La percepción se ubica dentro de los llamados procesos psicológicos básicos. Según Colmenero (2014) entendemos la "percepción como una compleja transformación de los efectos que los estímulos ejercen sobre nuestros sistemas sensoriales en información y conocimiento sobre los diferentes elementos, objetos y entidades de nuestro ambiente" (2014, pág. 46). A partir de esto, la percepción puede considerarse como uno de los procesos psicológicos más importantes, pues está en la base de nuestra capacidad para desenvolvernos en el mundo.

La definición de percepción ha ido evolucionando con el tiempo. Diversas disciplinas, como la Antropología, la Filosofía y la Psicología, han estudiado este tema. La percepción es el 
proceso cognitivo de la conciencia que consiste en el reconocimiento, interpretación y significación para la elaboración de juicios (Vargas, 2004, pág. 48). Debemos enfatizar que cuando hablamos de la percepción se involucran referentes ideológicos y culturales que reproducen y explican la realidad, de esta manera son aplicados a las diversas vivencias del día a día.

Este artículo presenta estudios previos relaciones al tema de la investigación, a continuación se describen los materiales y método empleado. Posteriormente se exponen los resultados y finalmente la discusión y las conclusiones.

\section{Estudios relacionados}

En primer lugar, uno de los estudios realizados acerca del tema es el de Arancibia, Cosimo y Casanova (2018). En este trabajo se hace referencia a una de las percepciones más recurrentes de los profesores del área de Lenguaje acerca del uso de TIC en el contexto escolar. Determinó que estas son fuente importante para la realización de clases y preparación de material. Señala, además, como resultado del estudio, la facilitación del aprendizaje gracias al uso de herramientas como videos, documentales, presentaciones power point. Estas permiten desarrollar clases "lúdicas" y "entretenidas". También mantienen la concentración e interés de los alumnos debido a la cercanía y naturalidad con que los nativos digitales utilizan estas herramientas tecnológicas en su vida cotidiana.

En segundo lugar, otro estudio realizado acerca de este tema es el publicado por la revista electrónica de investigación educativa titulado La percepción de la utilidad de la tecnología conforma su uso para enseñar y aprender. Este trabajo concluye que la tecnología es beneficiosa para alcanzar los objetivos de aprendizaje, la selección de los contenidos curriculares, la organización del tiempo y el espacio para el aprendizaje, y la mejora de la calidad del aprendizaje (Badia, Chumpitaz, Vargas y Súarez, 2016). Es importante aclarar que los participantes del estudio mencionado fueron docentes y los resultados no fueron correlacionados con la percepción de los estudiantes.

En tercer lugar, otro estudio realizado acerca de este tema es el titulado Uso de tecnologías de información en el aula (Jaramillo, 2005). En este trabajo participaron niños entre 8 y 11 años de edad. Las evidencias sugieren que, en general, los estudiantes al hacer uso del computador, no desarrollan argumentos en torno a algún tema. Tampoco reconocen la necesidad de la información para el aprendizaje. En ese mismo sentido, no adelantan actividades que les faciliten el desarrollo de productos para expresar su comprensión sobre algún tema.

En cuarto lugar, otro estudio realizado acerca de este tema es el titulado Percepciones de jóvenes acerca del uso de las tecnologías de información en el ámbito escolar (Silva, Borrero, y Marchant, 2006). En este se señala un resultado importante: alumnos y alumnas perciben que los profesores no valoran a las TIC en el proceso de enseñanza/aprendizaje escolar, por tal razón sienten que éstos le ponen restricciones al uso en las distintas asignaturas. Esta diferencia de visión hace que las tecnologías no se incorporen al proceso escolar y lleva a los jóvenes a establecer distintas estrategias para el uso de las TIC.

Los resultados de estas investigaciones aportan valiosa información. Los datos muestran las percepciones de docentes y, por otro lado, de estudiantes en el ámbito universitario. Nuestro estudio, por el contrario, brindará datos de niños en edad escolar y además se pretende categorizar las percepciones de los estudiantes acerca del uso educativo de dispositivos móviles y algo valedero es la posibilidad de que esta investigación permite relacionar la percepción del docente con la propia percepción del estudiante. 


\section{Materiales y método}

Este estudio se refiere al caso de una institución educativa de tipo regular y que ofrece los niveles de Educación General Básica. Está situada en el centro histórico del cantón Quito, capital de Ecuador. Su modalidad de estudios es presencial.

Esta investigación es de tipo cualitativo descriptivo. En cuanto al diseño es no experimental de tipo transversal descriptivo. Esto se debe a que el objetivo de este trabajo es examinar el impacto en la percepción de los estudiantes y docentes en cuanto al uso de las tabletas. El nivel de esta investigación es descriptivo ya que busca caracterizar el impacto del uso de las tabletas en docentes y estudiantes.

\subsection{Participantes}

Participaron los estudiantes de esta institución educativa que hacen uso de la tableta en su proceso de aprendizaje. La población está compuesta de 160 niños que cursan desde el segundo al séptimo año de Educación General Básica. La muestra se conformó con 70 niños, 10 docentes y autoridades de la comunidad educativa, quienes autorizaron el trabajo con la muestra mencionada.

\subsection{Normas éticas de investigación}

Se firmaron consentimientos informados con los participantes de la investigación (directivos de la institución, docentes y padres de familia). Los niños firmaron un asentamiento informado.

\subsection{Instrumentos}

En este estudio se realizaron entrevistas semi estructuradas con docentes de la institución educativa, y también se efectuaron grupos focales; sin embargo, se dio énfasis al trabajo de campo realizado con 70 niños. 21 cursan el cuarto año de educación general básica, 26 niños cursan el sexto de básica y 23 niños el séptimo de educación básica general. Los instrumentos utilizados fueron validados por profesionales en el campo de la Educación y la Psicología.

Los niños participantes de esta investigación hacen uso regular de las tabletas dentro del aula durante el abordaje de las diferentes asignaturas y contenidos de acuerdo con el currículo oficial vigente desde 2016. Estos niños colaboraron activamente en la actividad planteada que consistía en la escritura a mano de una carta donde se les solicitó expresar sus percepciones del uso de las tabletas. Los niños escribieron libremente con la consigna de que la carta sería leída por niños de la región amazónica del Ecuador que aún no conocían nada acerca de las tabletas y de su uso en el aula de clases.

\subsection{Técnicas de análisis de datos}

Se realizó una codificación de los datos que implicó la segmentación en temas para ubicarlos en las distintas categorías. En cuanto a estas categorías, se determinaron a partir del cuestionamiento analítico y la comparación mental de las distintas situaciones reflejadas en los datos. Parte del proceso incluyó un análisis intermedio de las cartas escritas por los niños para establecer las categorías que engloban la percepción de los niños frente al uso de las tabletas en el aula.

\subsection{Procedimiento}

Inicialmente se realizó una entrevista con la máxima autoridad de la Institución educativa para socializar los objetivos del proyecto de investigación y solicitar la autorización para iniciar el estudio. Posteriormente se realizó el grupo focal con los docentes que recibieron capacitación en el tema del uso de las tabletas en el aula, esto con la finalidad de detectar 
sus percepciones, temores y expectativas acerca del uso de un recurso digital durante el desarrollo de las clases.

Consecutivamente se realizaron entrevistas individuales a docentes con el objetivo de analizar el trabajo pedagógico y didáctico que ejecutan dentro del proceso de enseñanza y la percepción acerca del uso educativo de dispositivos móviles. En un siguiente paso, se trabajó con los niños con el fin de explorar sus sentidos, emociones, percepciones acerca del uso educativo de dispositivos móviles.

Posteriormente, se realizaron observaciones durante el trabajo en el aula y las diversas actividades propuestas fuera del aula de clase, específicamente en espacios recreativos. La información obtenida fue clasificada en categorías de acuerdo con las expresiones de los escolares.

\section{Resultados}

Se presentan los resultados obtenidos en la investigación en relación con el objetivo que implica "Determinar la percepción del docente acerca del uso educativo de dispositivos móviles".

En el grupo focal realizado previamente al uso de las tabletas en la institución educativa de estudio, los docentes plantearon ciertos temores entre los que se mencionaban:

- Miedo al posible daño de las tabletas.

- Reposición de los equipos en el caso de daño.

- Escasa capacitación en el software incluido en las tabletas.

- Compromiso de llevar a cabo el proyecto en la Institución.

Dentro de las expectativas de los docentes se puede detectar:

- Los recursos digitales son un instrumento para mejorar el aprendizaje.

- Los temores van a desaparecer con la práctica.

- Capacitación ampliada para poder replicar lo aprendido.

- Los niños llegarán a usar las tabletas mejor que los docentes.

- Los padres de familia aceptarán el uso de recursos digitales en el aula.

- El software es viable para generar otros programas. 
En las entrevistas individuales los docentes manifestaron sus experiencias en el uso de las tabletas a pesar de los temores expresados previo a la implementación del uso de recursos digitales. En la actualidad valoran positivamente el uso de la tecnología en el aula. Mencionan que los niños tienen mayor apertura, que la educación se vuelve más práctica, al manipular, ver, observar se les queda más grabado el aprendizaje, el aprendizaje es más profundo. También añaden que el uso de las tabletas es positivo porque es una herramienta que llama la atención a los niños y, porque les gusta, ponen "más atención". Además, los docentes se refieren a las tabletas como recursos motivadores que brindan a los niños la oportunidad de experimentar, sobresalir, de no limitarse, salir más allá.

A continuación, se presentan los resultados que dan cuenta del objetivo que implica determinar la percepción del estudiante acerca del uso educativo de dispositivos móviles. Igualmente, se observará la categorización de las percepciones de los estudiantes de acuerdo al nivel de educación general básica que cursan y, también, la relación de la percepción entre los actores educativos.

En el grupo de los niños de Cuarto de Educación General Básica, las respuestas acerca de su percepción al uso de las tabletas se clasificaron en las siguientes categorías:

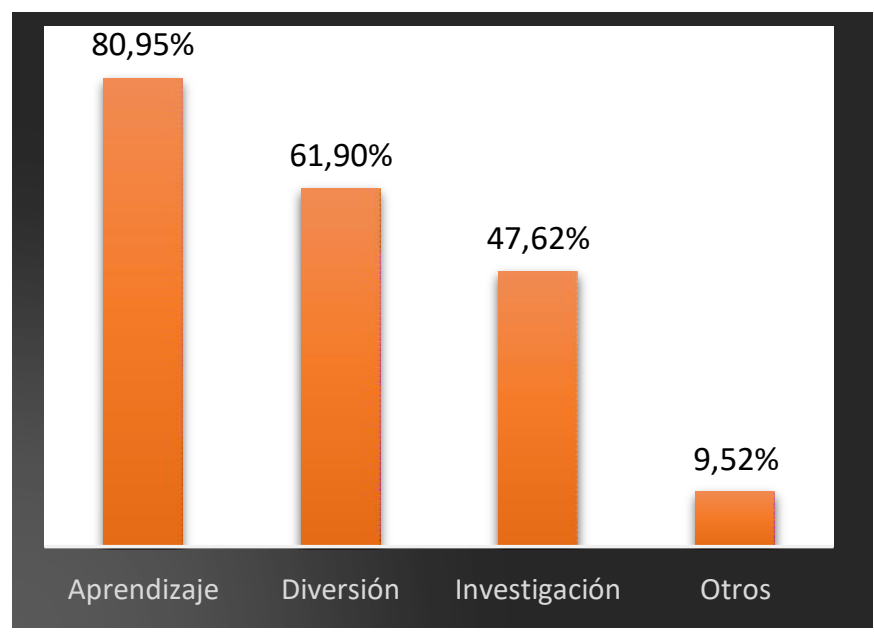

Figura 1. Categorías 4to. de Básica

Un aspecto destacable es la referencia que se hace acerca de que la tableta sirve para investigar. Es un indicador notablemente positivo puesto que las instituciones educativas deben propiciar el interés por indagar, consultar, descubrir e investigar, y precisamente esto es lo que refieren los niños. Es una evidencia de que la docente aplica "el aula inversa" en su proceso de enseñanza.

El aula invertida o flipped classroom es:

Un método de enseñanza cuyo principal objetivo es que el alumno asuma un rol mucho más activo en su proceso de aprendizaje que el que venía ocupando tradicionalmente. La idea principal de esta metodología está enmarcada en la posibilidad de que el alumno revise, estudie, se informe conceptos teóricos con el apoyo de diversos recursos o herramientas (Berenguer, 2016, pág. 1).

En la investigación realizada se evidencia que la docente participante motiva a sus estudiantes a revisar previamente en casa de los contenidos que serán abordados en clase. Los niños realizan esa revisión no únicamente en internet en vista de que no todos cuentan con este recurso. Pueden investigar a través de otros recursos: textos físicos, periódicos o 
información extraída de sus familiares. Llegan a la escuela con conocimientos previos. Además con la posibilidad de contar con un tiempo para revisar los contenidos a través de las tabletas. Este procedimiento les ayuda a lograr un aprendizaje significativo de acuerdo con lo expresado por la docente.

El aprendizaje significativo se basa en la siguiente idea: "el conocimiento verdadero solo puede nacer cuando los nuevos contenidos tienen un significado a la luz de los conocimientos que ya se tienen" (Ausubel, 2002, pág. 122).

En el grupo de los niños de Cuarto Año de Educación Básica de estudio se destaca la metodología de la docente como motivante y significativa en el tema de la investigación. Uno de los niños expresó por ejemplo, que trabajar con las tabletas era lo mejor que le pudo haber pasado en la escuela porque aprendía y se divertía mucho investigando con la tableta.

En el grupo de los niños de Sexto de Educación General Básica las respuestas se clasificaron en las siguientes categorías.

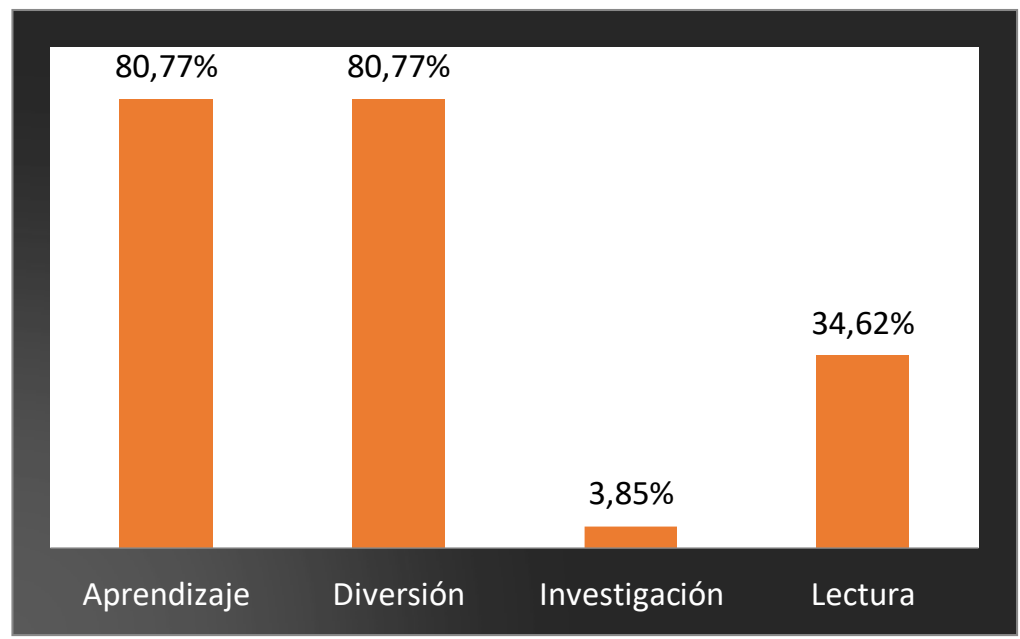

Figura 2. Categorías 6to. de Básica

Los resultados de las respuestas obtenidas en niños de sexto de educación básica guardan una relación directa con las respuestas emitidas por los niños de cuarto año de Básica en cuanto a las categorías. Sin embargo, el grupo de niños de Sexto de básica considera que el uso de las tabletas está alineada directamente con el aprendizaje y con la diversión. Estas dos categorías alcanzan exactamente el mismo porcentaje y un mínimo porcentaje considera a la tableta como un recurso para investigar. En este grupo se percibe otra categoría referida a la lectura.

Otro grupo de niños consultados expresa que la tableta sirve para leer, esto se relaciona directamente con lo referido por la docente responsable de este grupo de niños. En la entrevista efectuada mencionó que la tableta es un buen recurso para que los niños lean, sobre todo cuentos ya que considera que la información cargada en las tabletas es muy básica y deficiente. Por su parte, uno de los niños de este grupo expresó que utilizar las tabletas es "genial", es como utilizar un libro, pero también es posible encontrar cuentos, sopas de letras y muchas cosas más". 
En el grupo de los niños de Séptimo de Educación General Básica las respuestas que evidencian la percepción de los niños con respecto a la tableta se clasificaron en las siguientes categorías:

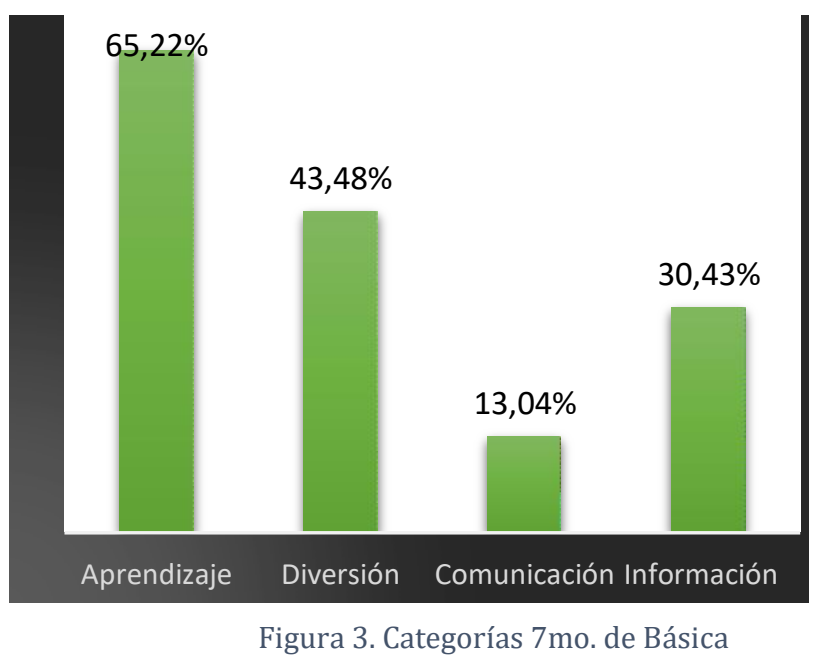

En el grupo de los niños de Séptimo de Básica aparecen dos nuevas categorías: Comunicación e información. La comunicación está relacionada con el uso de las redes sociales y la información con la posibilidad de usar la tableta como recurso para obtener información. Estas dos categorías no se evidenciaron en los grupos de estudio anteriores. En este punto se considera relevante mencionar que los niños de Séptimo año con una edad cronológica entre 11 y 12 años se benefician cuando hacen cosas con sus pares. En esta etapa "desarrollan habilidades necesarias para practicar la sociabilidad y la intimidad, lo que les permite tener un sentido de pertenencia" (Papalia, 2012, pág. 336). Esta característica de la etapa de desarrollo de este grupo de niños se relaciona con las ideas que mencionan acerca de la correlación entre el uso de las tabletas y las redes sociales. Los niños en esta edad "aprenden roles y reglas así como habilidades de liderazgo, cooperación y comunicación" (Papalia, 2012, pág. 336). Este último aspecto -"la comunicación"- vuelve a tener una implicación directa con las redes sociales, cabe aclarar que dentro del aula los docentes no permiten el uso de redes sociales, los recursos que se usan no están alineados a este tema, sin embargo, debe considerarse esta mirada que dan los niños de esta edad. Se debe destacar que los estudiantes de este grupo ratifican lo señalado por los niños de cuarto y sexto de EGB.

\section{Discusión y conclusiones}

Resultan significativos los resultados encontrados en este estudio, puesto que a nivel general se observa que un $75 \%$ de los niños participantes de esta investigación asocian el uso de la tableta con el aprendizaje. Mencionan que el recurso digital les permite acceder a información en áreas relacionadas con las asignaturas que forman parte del currículo de estudio. Añaden también que les sirve para "estudiar" refiriéndose a la preparación que realizan previo al rendimiento de las evaluaciones académicas.

Entonces, si las tabletas permiten aprender sería importante analizar las estrategias didácticas de los educadores, es decir, considerar la posibilidad de que las tabletas sirven también para enseñar pensando en que los recursos tecnológicos son un medio para llegar al conocimiento. Sin embargo, no hay que olvidar que éstos no constituyen un fin en sí mismo. Las metodologías usadas en el aula deben estar alineadas al mundo circundante y responder a los intereses de los estudiantes logrando una motivación al aprendizaje. 
Varios estudios han demostrado que la mejora del proceso de enseñanza-aprendizaje está vinculado directamente con el factor motivacional. Ospina (2006) afirmó que "uno de los aspectos más relevantes para que se dé el aprendizaje es la motivación y no hay duda alguna acerca de que cuando esta no existe, los estudiantes difícilmente aprenden" (pág. 158). Los estudiantes participantes del presente estudio mostraron indicios de encontrarse motivados frente al uso de las tabletas. Sus docentes también reportan aspectos positivos en las actitudes de los estudiantes que demostrarían un buen nivel de motivación frente al uso del dispositivo, esto facilitarían el aprendizaje.

Por otro lado, algo valorable es la percepción que los estudiantes expresan al referir la tableta como recurso óptimo para investigar. Esto se considera altamente positivo en vista de que desde las instituciones educativas se debe propiciar el interés por indagar, consultar, descubrir e investigar y precisamente esto es lo que refieren los niños.

Ossa (2005) en su estudio Educar es enseñar a indagar, la investigación como proceso de formación, afirma la necesidad de "desmitificar la investigación con el objetivo de erradicar el mito de que investigar es inalcanzable" (pág. 526). Los seres humanos podemos investigar en vista de que ser humano significa ser curioso. Ser indagador no es asunto de élites, es connatural al humano. Por ende, el propiciar la investigación en tempranas edades permite el pronto desarrollo de estrategias de adquisición científica.

Si la tableta es vista como un recurso para la investigación, hecho interiorizado en los estudiantes, entonces los docentes y el sistema educativo en general ya cuenta con una excelente herramienta, un capital que puede reportar acceso de calidad al conocimiento. Se requiere, en ese sentido, un fortalecimiento en el uso del recurso a fin de sostener y mejorar esa visión del estudiantado acerca de este recurso tecnológico.

En esa misma línea, los niños de cuarto año participantes de este estudio aseguran que la tableta les permite precisamente realizar actividades ligadas a la indagación y la investigación. Un dato relevante es que este resultado se relaciona directamente con la metodología empleada por la docente responsable del grupo, ya que da énfasis a la investigación, despertando el interés y la curiosidad por la indagación de temas relacionados a las áreas de currículo. La docente considera que un libro es limitante y que el internet y la tecnología permiten a los educandos ir más allá de lo establecido en el currículo ordinario, por ello a diario ejecuta la metodología del aula inversa permitiendo así un aprendizaje más dinámico, participativo con muchas posibilidades de interacción entre los estudiantes. El uso de la tableta para la docente es un recurso clave que permite la implementación de metodologías innovadoras en el aula.

Si pensamos como Leiva que asevera que para los docentes el origen de los conflictos que se producen en sus centros es sobre todo de índole social $(51,3 \%)$, podremos dar mayor valor a ese juicio emitido por los niños acerca de la tableta como vehículo de conocimiento a otras realidades. En esa misma investigación, un $23 \%$ de los docentes considera que el origen de los conflictos que surgen en sus escuelas es emocional. Un 15,4\% de los docentes valora el origen de los conflictos en la dimensión cultural, mientras que solamente un 10,3\% opinan que este origen se da en el ámbito propiamente académico, este resultado es relevante puesto que confirma una tendencia entre los docentes a concebir que los conflictos escolares tienen cada vez más una dimensión o vertiente social frente a la puramente académica (2008, pág. 5).

Por tanto, se concluye que la interculturalidad debe ser propuesta y trabajada en las instituciones educativas con el fin de generar actitudes que, partiendo del respeto a otras culturas, se supere las carencias del relativismo cultural. El respeto y la valoración de otras culturas llevará a un encuentro de convivencia, virtud escasa en nuestra sociedad. De 
acuerdo con la percepción de los estudiantes y los docentes, el uso de la tableta entonces permitiría conocer otras culturas a través de la búsqueda en programas como el google. Esto es posible gracias a que las tabletas del proyecto cuentan con conexión a internet. Esta actividad debería ser propuesta y guiada por el docente en vista de que los recursos instalados previamente en la tableta no incluyen ningún elemento que facilite este conocimiento.

La propuesta de usar la tableta en diversas actividades como se ha mencionado anteriormente requiere de la capacidad creativa e innovadora del docente. Sin embargo, exige también una capacitación sostenida y regular que además brinde a los docentes la seguridad de ser aptos para solventar los procesos en el aula.

Es relevante considerar además la predisposición hacia el uso de un recurso innovador. Antes del inicio de la ejecución del Proyecto Aula Digital, los docentes expresaron temores frente a algunos aspectos referidos especialmente a la poca capacitación recibida. En este sentido, sobre todo señalan la falta de práctica con el objeto tableta antes de su uso en el aula con los estudiantes.

Para algunos docentes la tableta podría estar relacionada con una amenaza en vista de que consideran que los niños son nativos digitales frente a los docentes catalogados por ellos mismos como inmigrantes digitales. El uso de la tableta y la familiaridad con la misma, bajó los niveles de temor frente al uso de un recurso digital desconocido para muchos, sobre todo para aquellos docentes que superan los 45 años de edad y llevan una larga trayectoria en el Magisterio ecuatoriano.

Los docentes además expresaron temor de un daño físico del aparato electrónico y se cuestionan acerca de cómo se realizaría la devolución de este en caso de producirse un daño ocasionado por el mismo docente o por uno de los estudiantes. Cabe recalcar que los docentes que se desempeñan en niveles de Educación Básica Elemental (segundo, tercer y cuarto año de básica) muestran más aprensión por el daño de los equipos en relación con los docentes del nivel Básica Media (quinto, sexto y séptimo de básica) y refieren este aspecto a que los niños en edades tempranas suelen tener menos cuidado de los recursos, así mismo indican que previamente al uso de las tabletas es conveniente dedicar un tiempo a la preparación de los estudiantes en el cuidado óptimo de los equipos. Estos aspectos evidencian la necesidad de capacitar a docentes y pensar en un trabajo previo a la implementación de Proyectos con los estudiantes, es trascendente determinar necesidades, realizar un adecuado diagnóstico y con base en esto planificar y ejecutar proyectos ligados a necesidades comunitarias, institucionales y personales.

Otro aspecto al que los niños hacen referencia es la posibilidad de aprender operaciones matemáticas, a través del uso de las tabletas, proceso de gran relevancia dentro de la educación general básica. En el presente estudio, los participantes muestran interés en el uso tecnológico vinculado al aprendizaje de la Matemática.

Otros niños también se refieren a un aprendizaje de valores por medio del uso de las tabletas, este aspecto se alinea con el Acuerdo Nacional anunciado por el Ministerio de Educación (MINEDUC, Vuelven a las aulas el maestro, los valores y la alegría, 2019) donde menciona precisamente que a través de la educación se van a recuperar valores. Por este motivo, incorporar recursos en las tabletas que apoyen el trabajo de Educación en valores encaminados al cumplimiento del desarrollo de capacidades ciudadanas optimizaría los resultados deseados en este actual acuerdo nacional referido. El discurso de la máxima autoridad del Ministerio de educación parece estar interiorizado por los docentes participantes de este estudio y por ende por los niños quienes suelen replicar no solo el discurso sino las actitudes de quienes consideran su modelo: el docente/autoridad. 
Otro aspecto que los niños participantes de este estudio mencionan es la posibilidad de leer cuentos e historias en este recurso digital. La lectura permite un aporte altamente significativo en el proceso de aprendizaje. Este aspecto ha sido considerado por el Ministerio de Educación que asegura que "la ausencia de la actividad de lectura es una de las grandes debilidades en la construcción del sentido de ciudadanía en el Ecuador" (MINEDUC, Textos escolares y lectura en el sistema educativo, 2018).

Por consiguiente, el Ministerio de Educación promueve proyectos que buscan fortalecer la motivación y el gusto por la lectura en toda la comunidad educativa no únicamente en los estudiantes. De hecho un portal web del mismo Ministerio se señala que "si los profesores no leen, difícilmente podremos tener estudiantes que lo hagan". En consecuencia si el uso de la tableta en el aula permite el acercamiento y la posibilidad a la lectura este proceso debe fortalecerse y alinearse con otros proyectos gubernamentales como el "Plan Nacional de Promoción del Libro y la Lectura del Ecuador que se fundamenta en una política pública que abarca los principales problemas relativos al estímulo de la lectura en el Ecuador" (Ministerio, 2019, pág. 4). Este proyecto además determina la necesidad de fomentar los comportamientos lectores y los consumos de lectura para promover una sociedad equitativa y soberana, como lo determina la Ley Orgánica de Cultura en sus artículos 120 y 126. El cumplimiento de este mandato constitucional es competencia del Ministerio de Cultura y Patrimonio y del Gobierno Nacional" (Ministerio, 2019, pág. 4).

Si bien es cierto que la ejecución de este proyecto es competencia del Ministerio de Cultura y Patrimonio, se debe unificar esfuerzos entre dicho Ministerio con el Ministerio de Educación sumado a la empresa privada -como es el caso de Fundación Telefónica- de tal forma que los resultados, en ámbito de procesos lectores, tengan una incidencia significativa en los procesos de enseñanza aprendizaje. Si los niños perciben y valoran la tableta como un recurso óptimo para leer y lo descubren como si fuera igual a un libro, lo ideal sería propiciar que dentro del plan lector propuesto por el Ministerio de Educación se propongan actividades articuladas con el uso de las tabletas en todos los niveles educativos, fomentando además la lectura en los docentes.

Para finalizar la discusión en relación con la apreciación de los niños acerca de la tableta como recurso educativo, se puede concluir con la idea de la importancia de un aprendizaje divertido o, por su contraparte, la diversión llevada a un aprendizaje significativo. De hecho, todo proceso educativo debería ser percibido por un niño como un espacio de diversión que permita aprender para la vida.

En la aplicación de las tabletas como recurso dentro del aula se ha podido comprender en este estudio que la guía, percepción, valoración y aceptación del recurso digital por parte del docente incide directa y considerablemente en la percepción de los estudiantes.

Por consiguiente, el rol del docente en el aula a través del uso de la tableta incidirá considerablemente en la percepción, uso, utilidad y valoración que sus estudiantes tengan del recurso digital encaminado todo esto hacia una motivación por el aprendizaje. Por ello, se requiere de una capacitación docente sostenida con procesos de seguimiento y evaluación que permitan reajustes en la ejecución del Proyecto Aula Digital así como el uso de otras metodologías innovadoras en el aula.

Es claro que la tableta como recurso digital dentro del aula ha logrado resultados positivos en los procesos de aprendizaje. La totalidad de los participantes perciben y valoran el recurso como positivo. Lo asumen como un puente que les acerca al conocimiento de otras culturas. Les da la posibilidad de investigar generando un aprendizaje significativo.

Esta investigación abre el camino a indagar acerca del mejoramiento en el rendimiento académico de los estudiantes a partir del uso de las tabletas.

Licencia Creative Commons Atribución 4.0 Internacional (CC BY 4.0)

Revista Cátedra, 2(3), pp. 39-53, septiembre-diciembre 2019. e-ISSN: 2631-2875

https://doi.org/10.29166/catedra.v2i3.1833 


\section{Bibliografía}

Arancibia, M., Cosimo, D., \& R, C. (2018). Percepción de los profesores sobre integración de TIC en las prácticas de enseñanza en relación a los marcos normativos para la profesión docente. Ensaio: aval. pol. públ. Educ, 26(987), 163-184,.

Ausubel, D. (2002). Adquisición y retención del conocimiento: una perspectiva cognitiva. Barcelona: Paidós.

Badia, A., Chumpitaz, L., Vargas, J., \& Súarez, G. (2016). La percepción de la utilidad de la tecnología conforma su uso para enseñar y aprender. Revista Iberoamericana de educación, 18(3).

Berenguer, C. (2016). Acerca de la utilidad del aula invertida o flipped classroom. Alicante: Universitat d' ALacant.

Colmenero, J. (2014). Procesos psicológicos básicos. Madrid: MC Graw Hill.

Fundación Telefónica. (11 de Octubre de 2017). Fundación Telefónica. Recuperado el 5 de Febrero de 2019, de ProFuturo llega a Ecuador para innovar la educación: http://fundaciontelefonica.com.ec

Fundación, T. (10 de 01 de 2018). Telefónica, Fundación. Recuperado el 31 de 01 de 2019, de Profuturo:http://fundaciontelefonica.com.ec/innovacion-educativa/profuturo/

Jaramillo, P. (2005). Uso de tecnologías de información en el aula. Revista de Estudios Sociales(20), 27-44.

Leiva, J. (2008). Interculturalidad, gestión de la convivencia y diversidad cultural en la escuela: un estudio de las actitudes del profesorado. Revista Iberoamericana de Educación, 46(2), 1-14.

MINEDUC. (16 de Mayo de 2018). Textos escolares y lectura en el sistema educativo. Recuperado el 6 de Febrero de 2019, de Textos escolares y lectura en el sistema educativo: https://educacion.gob.ec/textos-escolares-y-lectura-en-el-sistemaeducativo/

MINEDUC. (23 de 06 de 2019). Ministerio de Educación. Recuperado el 31 de Enero de 2019, de Memoria. Festival de la Innovación educativa.: https://educacion.gob.ec/memorias-documentos/

MINEDUC. (31 de Enero de 2019). Vuelven a las aulas el maestro, los valores y la alegría. Recuperado el 6 de Febrero de 2019, de Vuelven a las aulas el maestro, los valores y la alegría: https://educacion.gob.ec/vuelven-a-las-aulas-el-maestro-los-valoresy-la-alegria/

Ministerio, C. y. (6 de Febrero de 2019). Plan Nacional del Libro y la Lectura José de la Cuadra. Recuperado el 6 de Febrero de 2019, de Plan Nacional del Libro y la Lectura José de la Cuadra: http://oei.org.ar/new/wp-content/uploads/2018/03/PNPLLEcuador.pdf

Ospina, J. (2006). La motivación, motor del aprendizaje. Ciencias Salud, 158-160.

Ossa, J. (2005). Educar es enseñar a indagar. La investigación como procesos de formación. Educación, XXVIII(3), 525-533.

Papalia, F. (2012). Desarrollo Humano. México: Mc Graw Hill. 
Silva, I., Borrero, A., \& Marchant, P. (2006). Percepciones de jóvenes acerca del uso de las tecnologías. Juventud, educación, 14(24).

Vargas, L. (2004). Sobre el concepto de la percepción. Alteridades, 4(8), 47-53. 


\section{Agradecimiento}

Se agradece a Fundación Telefónica y el equipo de su proyecto ProFuturo. Asimismo, la colaboración interinstitucional requerida para presentar este trabajo fue posible gracias al Ministerio de Educación de la República del Ecuador dentro de la Agenda Educativa Digital 2018-2021. Finalmente se extiende el reconocimiento al equipo de investigación de PUCE "Proyecto Aulas Digitales Fundación Telefónica", conformado por: Balladares Burgos Jorge, Cruz Silva Jorge, Egas Reyes Verónica, Loza Aguirre Edison, Maldonado Garcés Verónica, Miranda Orrego María Isabel, Rivas Toledo Alexis, Roa Marín Henry y Salao Sterckx Emilio. Los estudiantes, Grijalva Alvear Isaac, León Bayas Cristina, Terán Paz y Miño Daniela, Rivadeneira Yépez Ana Dominique.

\section{Autores}

Verónica Maldonado Garcés obtuvo su título de Magíster en Educación Infantil y Educación Especial con doble titulación en la Universidad de Cádiz y la Universidad Tecnológica Equinoccial (2007). Obtuvo el título de Psicóloga Educativa en la Universidad Politécnica Salesiana.

Actualmente es docente e investigadora de la Pontificia Universidad Católica del Ecuador. Su línea de investigación abarca los procesos psicológicos y educativos, así como temas de discapacidad, inclusión y diversidad.

Jorge Balladares Burgos obtuvo su título de Doctor en Formación del Profesorado y TIC en Educación en la Universidad de Extremadura (España) en el 2017, y logró el Premio Extraordinario de Doctorado 2016/2017 otorgado por el Consejo de Gobierno de la Universidad de Extremadura. Obtuvo el título Magíster en Tecnologías aplicadas a la gestión y práctica docente en el año 2012, y el título de Magíster en Filosofía en el año 2005 en la Pontificia Universidad Católica del Ecuador. Obtuvo el título de Licenciado en Filosofía en el año 1997 y el título de Profesor de la enseñanza media y especial en Filosofía en el año 1996 en la Universidad del Salvador (Argentina).

Actualmente es profesor en la Universidad Simón Bolívar y en la Pontificia Universidad Católica del Ecuador. Sus principales temas de investigación incluyen la educación digital, la formación del profesorado, TIC aplicadas a la educación, educación en línea, híbrida, móvil y disruptiva, innovación educativa, políticas educativas públicas; etnofilosofía, ética digital, inclusión educativa digital y humanismo digital.

Alexis Rivas Toledo. Doctor en Ecología, Universidad Autónoma de Madrid, España; Maestro en Antropología Social por el Centro de Investigaciones y Estudios Superiores en Antropología Social-CIESAS de México D.F., México; Antropólogo Social, Pontificia U. Católica del Ecuador-PUCE, Quito.

Es profesor investigador especializado en sistemas políticos, etnicidad, democracia, sostenibilidad ecológica, salud y antropología médica. Estudioso del rol de las ONG y la sociedad civil de frente a la construcción de movimientos sociales, identidades étnicas, políticas públicas y democratización. Ha sido profesor invitado de la Escuela Superior Politécnica del Litoral-ESPOL, Guayaquil, Ecuador, FLACSO Sede Ecuador, Universidad Internacional de Andalucía-UIA, España. Es docente en F. Medicina-PUCE. 